\title{
The Influence of Sudarshan Kriya Yoga on Sleep Quality in Indian Adults: An Open Trial Pilot Study
}

\author{
Divya Kanchibhotla $^{1}$ [ $\cdot$ Sheel Galada Parekh ${ }^{1} \cdot$ Prateek Harsora $^{1} \cdot$ Shashank Kulkarni ${ }^{1}$
}

Received: 19 December 2020 / Revised: 14 April 2021 / Accepted: 17 May 2021 / Published online: 30 May 2021

(c) The Author(s) 2021

\begin{abstract}
Purpose Sudarshan Kriya Yoga (SKY) is an advanced yogic breathing technique with a demonstrated impact on human physical and mental health. Even so, very few studies have observed its effect on sleep. This is the first study evaluating the impact of SKY as an intervention on sleep quality among Indian population. This study assessed both the immediate and prolonged effect of SKY on sleep quality. The secondary objective of the study was to evaluate the association between frequency of SKY practice and sleep quality.

Methods This was a single arm open-trial study, which included 473 participants. All participants underwent a 3-day SKY workshop offered by the Art of Living, and were assessed for sleep quality using the Pittsburgh Sleep Quality Index (PSQI) questionnaire, administered to the participants before the program (pre-intervention), immediately after the program (postintervention), and at Day 40 (D40) after the program. Responses were grouped according to age, gender, and frequency of practice. The impact of the frequency of SKY practice on sleep quality was also investigated.

Results The quality of sleep improved with SKY practice across the study population. Women recorded inferior quality of sleep at the beginning of the study, but experienced a greater improvement in sleep quality after the SKY practice, as compared to men. The younger population seemed to benefit over the long-term, while the older population had an immediate improvement in sleep quality, however, they were not able to sustain it. The effect size varied with the frequency of practice. Sleep quality improved markedly among those who practiced SKY daily, indicating an association between the frequency of SKY practice and its efficiency in improving sleep quality.

Conclusion The results demonstrate the efficacy of SKY in improving sleep quality across populations, irrespective of gender or age. This is indicative of the beneficial role of SKY in treating psychological disorders such as insomnia. The effect varies with the frequency of practice, with more frequent practice yielding better quality of sleep.
\end{abstract}

Keywords Sudarshan Kriya Yoga (SKY) $\cdot$ Yogic breathing $\cdot$ Sleep quality $\cdot$ Insomnia

\section{Introduction}

Sleep is one of the important self-regulatory functions of the body, vital in clearing brain waste, maintaining brain function, energy conservation and modulating the body's immune response. It was found that sleep is regulated globally, regionally, and locally, by means of both molecular and cellular mechanisms [1]. There is a bidirectional relationship between sleep and health. While sleep disturbances lead to

Divya Kanchibhotla

director.ssiar@artofliving.org

1 Sri Sri Institute for Advanced Research, 21st Km Kanakapura Rd, Udayapura, Bengaluru, Karnataka 560082, India the development of various medical and psychiatric disorders, the same disorders in turn, result in poor sleep quality [2-4]. The problems associated with sleep disturbance and sleep deprivation are many, including not just anxiety and depression, but also the risk of obesity, hypertension, diabetes, cardiovascular diseases, metabolic disorders, respiratory disorders, insomnia, sleep apnea, chronic pain, and even cancer $[4,5]$. The prevalence of insomnia and sleep apnea increases with age and poor sleep quality is a common problem found amongst older adults [3, 4].

Just like sleep, breathing is also a vital physiological function, and crucial to our existence. There has been an increased interest in breathing techniques as a way to enhance emotional regulation, mental well-being and physical health. Several research studies demonstrate that 
different breathing patterns are connected with unique emotions and physiological conditions. Breathing patterns change in response to emotional or physiological conditions: for example when experiencing anger, or during exercise, breathing rate increases. Respiration is key in maintaining physiological homeostasis and co-exists with emotions [6]. In a systematic review, evidence was found to substantiate a link between parasympathetic activities, CNS (Central Nervous System) activities related to emotional control and psychological well-being, in healthy subjects during the practice of a slow breathing technique [7].

One breathing technique that is demonstrably efficacious in improving psychological states and physiological conditions in adults is an advanced breathing technique known as Sudarshan Kriya Yoga (SKY). The universal effect of SKY is to promote calm and a sense of well-being. Research on SKY technique demonstrates its efficacy in decreasing stress, anxiety and depression [8-14], while mitigating the effect of negative experiences $[15,16]$. It increases emotional regulation [17], decreases impulsiveness while increasing mental focus $[18,19]$, provides a sense of calm and enhances wellbeing [8, 20,21]. It is known to improve cardiac metrics [20, $22,23]$ and vagal tone [21, 24-28].

Several studies have documented the benefits of SKY on physical and mental health, however, little is known about its effects on sleep specifically. Previously, a cross sectional study examined the effect of SKY on sleep quality among experienced practitioners, and another study assessed its impact on sleep architecture. The study on sleep architecture found that although the sleep architecture was comparable among the younger control and yoga groups, slow wave sleep [non-REM (rapid eye movement) S3 and S4] had reduced in the middle aged control group. Participants of the middle-aged SKY group showed no such decline in slow wave sleep states [29].

There are several mind-body practices and interventions such as yoga [30, 31] and cognitive behavioral therapies (CBT) [32], which improve the quality and duration of sleep. A large body of research has been devoted to investigating the effects of meditation techniques like mindfulness meditation or transcendental meditation on sleep [33-35]. The immediate beneficial role that SKY might have on sleep is understudied. This research study is a step towards addressing this gap in the available literature. This is the first study on SKY which investigates its immediate and prolonged effect on sleep quality through an open-trial pre-post study design. Our hypothesis being that the SKY practice will improve the sleep quality and its effect will last for longer duration with practice. This study explores the changes in sleep quality among general adult population with SKY, using a self-report questionnaire at three different time points, prior to the intervention (Pre), just after the intervention (Post), and after 40 days of practice (D40). The study design allows for further investigation into the longterm effects of SKY on sleep, and the association between the frequency of SKY practice with sleep quality. To our knowledge, this is one of the first study that associates the long-term efficacy of a yoga-based intervention with the frequency of its practice.

SKY is an advanced breathing practice. It is a composite technique with three pranayamas and unique rhythmic breathing patterns involving fast, medium and slow breaths. It is widely popular and numerous research studies have demonstrated its efficacy, hence we found it crucial to study the impact of this highly replicable and widely used technique on sleep. Regular practice of SKY can bring solace to millions looking for improved sleep quality.

\section{Methods}

\subsection{Study Design}

An open-trial single arm pre-post study design was adopted for this study. The data were collected using a self-report questionnaire from the participants enrolled for the Happiness Program (SKY workshop) at The Art of Living International Center, Bangalore. A standardized questionnaire that measured the quality of sleep for an individual was used for assessment. The assessment was conducted at three different time points: before the intervention, i.e., at the beginning of the first day of the workshop (Pre), immediately after the workshop (Post), and lastly after the recommended 40-day daily practice of the Sudarshan Kriya Yoga (Day 40).

\subsection{Population}

The study was conducted at The Art of Living International Center Bangalore, India, between August 2018 and August 2019. The Happiness Program (SKY workshop) was conducted at the center each weekend from Friday to Sunday. Participants included adults above 18 years of age. Both genders were included in this study. The educational and economic backgrounds varied among the study population, as did dietary habits. All those who had registered for the Happiness Program, were notified about the study in detail on the very first day, before the first session of the program. Participation was sought only from those who had no previous experience of SKY. Those who agreed to participate in this study were recruited with their informed consent. The Institutional Review Board of the Sri Sri Institute for Advanced Research in India approved this study.

Participants were trained in SKY over 3-days. During the program, all the participants were provided "Home Going instructions" which enabled them to practice SKY independently at home. On the last day of the program, they 
practiced SKY on their own with the instructor on site, to ensure that they were practicing the technique correctly. In addition, daily follow-up sessions were conducted and weekly follow-up sessions were provided, where participants practiced along with the teacher and could clarify any questions or doubts. During these sessions, instructors were also able to guide them and ensure they were practicing correctly. Participants were encouraged to attend the daily and weekly sessions. Attendance logs were maintained. Online support tools and an app was also provided to the participants with instructions for the pranayamas and SKY to facilitate their practice. Weekly phone calls were made to enhance retention.

The study questionnaire was filled by the participants before the commencement of the program. Their personal details were kept confidential. On the last day of the program, the same set of questionnaires were once again completed by them. After 40 days of the completion of the program, subjects were requested through email and phone to complete the last stage of the study assessment. During the 40 day assessment, subjects were asked the following question "How frequently have you been practicing Sudarshan Kriya? They could respond as: Daily; 2-3 times a week or Once a week. Based on their response and maintained attendance logs, the long term effect of SKY practice on sleep was calculated.

A total of 473 people participated in the Pre and Post evaluation, while 112 subjects participated in Day 40 assessment.

\subsection{Intervention}

The Happiness Program is a 3-day life-skills workshop focusing on body, breath, and mental wellness. The core of the program includes a yogic breathing technique called the Sudarshan Kriya Yoga (SKY), along with interpersonal processes and activities. Trained teachers of The Art of Living facilitate the workshop, guiding participants through the SKY process, which takes an hour and 15 min. A shorter form of SKY, $35 \mathrm{~min}$, is also taught to the participants for daily home practice. For the study, participants were encouraged to practice the shorter version individually for 40 days after the commencement of the workshop.

A typical SKY session consists of four stages, each with its own distinct yogic breathing pattern, done while sitting in various postures (Ujjayi, Bhastrika, Om, and Sudarshan Kriya), interspersed with normal breathing. The first type, Ujjayi, involves deep, long breaths with attention to the sensation of the breath touching the throat. The pace is slow, at the rate of 2-4 breaths per minute (bpm). The second, Bhastrika, involves rapid and forceful exhalation at the rate of 25-30 bpm. This is followed by saying Om thrice, with prolonged expiration. The last stage, Sudarshan Kriya, involves rhythmic breathing at three different paces-slow breaths (15-20 bpm), medium (40-50 bpm), and fast (60-80 bpm). The entire sequence is done in a seated posture, with eyes closed. The aim is to elicit a mind-body response of quiet, calm alertness, experienced as a tranquil state in the rest period immediately following the practice.

\subsection{Study Measure}

The Pittsburgh Sleep Quality Index (PSQI) was used to collect data for the study. It is a standardized self-report sleep questionnaire that has been used across multiple populations in many languages. Consisting of 19 individual questions, it is a broad subjective measure that attempts to assess and measure seven components of sleep, including sleep quality, sleep latency (the time taken to fall asleep), duration of sleep, habitual sleep efficiency, sleep disturbances, use of sleep medication, and daytime dysfunction. Each component is based on a 0-3 Likert scale, and the component scores are aggregated to get a global PSQI score. A global PSQI score of 5 or more indicates poor sleep quality.

PSQI is one of the oldest scales, widely used in studies on yoga in India, with good reliability and sensitivity. Most of the items of PSQI are organized as multiple choice questions and are brief and easy to understand and answer. PSQI scale takes only 5-10 min to complete, and hence, was found appropriate for this study (Fig. 1).

\subsection{Data Analysis}

Participants were assessed at three-time points Pre, Post, and Day 40. The data were matched using their email addresses and phone number. The responses were then grouped by age, gender, and frequency of SKY practice. The mean score and Standard Deviation (SD) were calculated for each combination. The paired-sample $t$-test was used to compare differences between Pre and Post assessments, and Pre and Day 40 assessment. Validated software MS Excel 2007 (which has required statistical tests/functions) and IBM SPSS V25 were used for statistical analysis. In addition, Cronbach's alpha was calculated to measure the internal consistency and reliability of the data. Cohen's $d$ value was calculated to determine the effect size. Difference in mean value and pair-wise comparison was calculated using one-way ANOVA test, at 0.05 significance level. Since the data collected were on an ordinal scale, the following formula was used to calculate maximum sample size for a given $\%$ of error of estimate.

$n=0.25 \times Z^{2} / E^{2}$ where $Z=1.96$ for confidence level of $95 \%$ and $E$ is error of estimate.

To reduce the effect of Type 1 error due to multiple comparisons, Bonferroni correction was applied wherever possible. 
Fig. 1 Schematic presentation
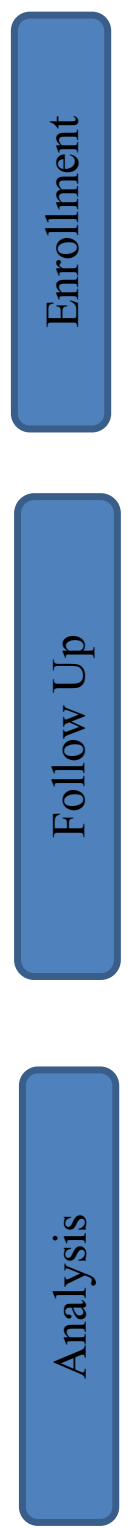

Statistical Analysis was performed in Groups (Age,

Gender \& Frequency of Practice) $(\mathrm{N}=473)$
SKY Practitioners

$(\mathrm{N}=27)$ were excluded
Post Assessment $(\mathrm{N}=473)$

Discontinued/Withdraw/Dro pouts $(\mathrm{N}=0)$
Table 1 Demographic details of participants

Participants characteristics $(N=473)$

\begin{tabular}{llc}
\hline Characteristics & Type & All participants \\
\hline Gender $n(\%)$ & Male & $266(56.24)$ \\
& Female & $207(43.76)$ \\
Age & Mean (SD) & $36.4(14.22)$ \\
\hline
\end{tabular}

\section{Results}

Table 1 depicts the demographic characteristics of all participants at baseline. Of the 473 participants, $56.24 \%$ were male and $43.76 \%$ were female, with average age of
36.4 years. Internal reliability was tested using Cronbach's alpha which was found to be $0.4,0.5$ and 0.6 , respectively, for all three time points.

Table 2 depicts a detailed distribution of global PSQI mean scores along with standard deviation and $p$ values, at three assessment time points, Pre, Post, and Day 40. For the overall population, the global PSQI mean score reduced significantly from 6.48 to 4.52 post the SKY workshop $(p<0.001)$. It increased slightly to 4.96 after 40 days of practice, but still remained significantly lower than the preintervention values.

Both genders experienced improved quality of sleep with the SKY practice. Women in the study had inferior quality of sleep to begin with and experienced a very significant improvement with SKY practice. The younger population 
Table 2 Mean scores and standard deviation for global PSQI with $p$ values at three different time points of assessment

\begin{tabular}{llllcc}
\hline Population & Pre mean (SD) & Post mean (SD) & Day 40 mean (SD) & $p$ value (pre-post) & $p$ value (pre-day 40) \\
\hline Overall & $6.48(3.84)$ & $4.52(2.94)$ & $4.96(2.76)$ & $<0.001^{* *}$ & $<0.001^{* *}$ \\
Male & $5.98(3.8)$ & $4.22(2.73)$ & $4.37(2.33)$ & $<0.001^{* *}$ & 0.006 \\
Female & $7.13(3.82)$ & $4.92(3.17)$ & $5.83(3.15)$ & $<0.001^{* *}$ & $0.001^{*}$ \\
Age 18-35 yrs & $6.76(3.83)$ & $4.33(2.71)$ & $4.55(2.57)$ & 0.006 & $<0.001^{* *}$ \\
Age 36+ & $6.09(3.83)$ & $4.81(3.23)$ & $6.05(3.11)$ & $<0.001^{* *}$ & 0.077 \\
\hline
\end{tabular}

$p$ values are based on one way ANOVA test at the significance level of $0.05,{ }^{*} p$ values $<0.005$ and ** $p$ value $<0.001$, PSQI is Pittsburgh Sleep Quality Index. A global PSQI score $<5$ is the indicator for good sleep quality. Pre: before SKY practice, Post: immediately after SKY practice, Day 40: after 40 days of SKY practice

Table 3 Percentage of population with good sleep quality (global PSQI score $<5$ )

\begin{tabular}{llll}
\hline Population & Pre $(\%)$ & Post $(\%)$ & Day $40(\%)$ \\
\hline Overall & 30.44 & 55.39 & 52.94 \\
Male & 34.96 & 59.4 & 66.67 \\
Female & 24.64 & 50.24 & 37.5 \\
Age-18-35 yrs & 27.01 & 56.57 & 55.88 \\
Age-36+yrs & 35.18 & 53.27 & 47.06 \\
\hline
\end{tabular}

PSQI is Pittsburgh Sleep Quality Index. A PSQI global score $<5$ is the benchmark for good sleep quality. Pre: before SKY practice, Post: immediately after SKY practice, Day 40: after 40 days of SKY practice

seemed to benefit with long-term practice of SKY while the older population had an immediate improvement in quality of sleep but was not able to sustain it.

Table 3 provides details of the percentage of study population with good sleep quality (PSQI $<5$ ) at the three different time points-Pre, Post, and Day 40. Both genders and both age-groups showed an increase in the percentage of population experiencing good sleep quality. The percentage of the overall population with global PSQI scores $<5$ jumped from 30.44 (Pre) to 55.39 (Post) and showed a slight decrease at the Day 40 assessment.
To further understand the results, and to investigate an association between frequency of practice and sleep quality, the population was divided in three categories: those who practiced SKY daily, those who practiced 2-3 times a week, and those who practiced only once a week. Table 4 depicts the Global PSQI values and percentage of population with PSQI scores $<5$ at pre-intervention and Day 40 for total population and divided by the frequency of practice. As can be seen from the table, the group that practiced SKY daily outperformed all the other groups (total population, other frequencies of practices) for the Global PSQI scores and the percentage of population with good sleep quality.

Effect size was calculated using Cohen's $d$ value. The effect size of the intervention was 0.68 for pre-post and 0.61 for pre-day 40 . This indicated a medium to large effect size. According to Coe et al. such an effect size signifies that $76 \%$ of the population experienced significant improvement in their sleep quality with the SKY intervention immediately, and $73 \%$ after 40 days of practice, respectively. As seen in Table 5, the effect size varied with the frequency of practice, decreasing as the frequency of practice went down. Table 5 lists the effect size.

Table 4 Association of frequency of SKY practice with global PSQI scores-[mean (SD) and $p$ values] and good sleep quality

\begin{tabular}{llll}
\hline & $\begin{array}{l}\text { Global PSQI scores } \\
\text { mean (SD) }\end{array}$ & $\begin{array}{l}p \text { value (pre-day 40 global } \begin{array}{l}\% \text { of popula- } \\
\text { PSQI values) }\end{array} \\
\begin{array}{l}\text { tion with PSQI } \\
\text { score }<5\end{array}\end{array}$ & 30.44 \\
\hline Total population-pre assessment & $6.48(3.84)$ & NA & 52.94 \\
Total population-day 40 assessment & $4.96(2.76)$ & $<0.001^{* *}$ & 56 \\
Frequency of SKY practice: daily-day 40 assessment & $4.60(2.57)$ & $<0.001^{* *}$ & 50 \\
Frequency of SKY practice: 2-3 times/week—day 40 assessment & $5.08(2.61)$ & 0.049 & 28.57 \\
Frequency of SKY practice: once/week-day 40 assessment & $5.86(3.42)$ & 0.066 & 5 \\
\hline
\end{tabular}

$p$ values are based on paired- $t$ test at the significance level of $0.05, * p$ values $<0.005$ and ${ }^{*} p$ value $<0.001$, PSQI is Pittsburgh Sleep Quality Index. A PSQI global score $<5$ is the indicator for good sleep quality. pre: before SKY practice, post: immediately after SKY practice, day 40: after 40 days of SKY practice 
Table 5 Effect size between different time points and for different frequency of practice of SKY

\begin{tabular}{ll}
\hline Population & $d$ value \\
\hline Pre-post & 0.68 \\
Pre-day 40 (overall) & 0.61 \\
Pre-day 40-daily & 0.65 \\
Pre-day 40-2-3/week & 0.60 \\
Pre-day 40-once/week & 0.50 \\
\hline
\end{tabular}

Pre: before SKY practice, post: after SKY practice, day 40 (overall): after 40-days practice of SKY including all subjects with any number of days SKY practice, day 40-daily: daily SKY practice for 40 days after commencement of workshop, day 40-2-3/week: practiced SKY 2-3 times weekly for 40 days after commencement of workshop, day 40-once/week: practicing SKY once a week for 40 days after commencement of workshop

\section{Discussion}

SKY is a yogic technique which enhances subjective feelings of well-being, while reducing anxiety and depression, as well as creating a positive impact on various other physiological and psychological conditions. A study that investigated the impact of SKY on sleep, suggested that REM onset latency in a middle-aged group of regular SKY practitioners is short, while slow-wave sleep is similar to that of a younger control group [29]. There are only a few studies that have originated from India on Yoga and Sleep, especially on healthy adults, and even fewer studies have tracked long term effects. This is the first study to observe effects of SKY on sleep, including the association between the frequency of practice and effect on sleep. We hypothesized that SKY might have positive impact on sleep quality, and focused exclusively on examining the immediate and residual long-term effects of this distinctive breathing technique in a healthy population.

Our results indicate a significant improvement in the sleep quality of study population with SKY. SKY practice appears to benefit both male and female populations significantly. Women appeared to have a poorer quality of sleep to begin with, and there was a significant improvement at the end of day 3. The younger age group, who mostly reported poor quality of sleep before the intervention, seemed to benefit more from the practice. The restorative sleep pattern persists with frequency of practice, particularly in the younger population.

The results show that immediately after the Happiness Program, the percentage of overall population with good quality of sleep (PSQI <5) almost doubled, which is a substantial difference. At day 40, although the percentage of people reporting good sleep quality fell slightly, there continued to be a substantial impact, with global PSQI scores remaining significantly lesser than pre-intervention scores. Based on the gender and age analysis, it can be inferred that this restorative sleep pattern persists over an extended period of time across populations, especially with frequency of practice, particularly in the younger population.

Review of existing research on the impact of meditation and related techniques on sleep issues $[5,36]$ indicate that meditation has a regulatory role on sleep, and even at 5- to 12-month follow-up, the effect of mindfulness meditation is not different from evidence-based sleep treatments, and sleep quality improves appreciably compared to control group [33]. The current study, while focusing on the specific technique (SKY), adds to the existing research by demonstrating that a positive impact on sleep can also be achieved by using breathing based practices. This study is a valuable addition to prior research on SKY and mind body interventions in general, which reveal that the technique can benefit a variety of populations in multiple positive ways [8-23, 29, 37]. A recent study compared SKY with a university course called Foundations in Emotional Intelligence (EI) and a Mindfulness-Based Stress Reduction (MBSR) program on a student population, found that SKY had a better impact the EI or MBSR. The comprehensive, multi-component nature of SKY practice makes it a valuable practice: one aspect that, according to the researchers, warranted further study was its impact on sleep [19].

As observed in our study, regular practice of SKY is important to acquire sustainable and improved sleep quality. Reduction in mental stress and improvement in cognitive performance after SKY was observed with the help of EEG (Electroencephalograph), ECG (Electrocardiogram) and DT (Determination Test) [26]. A single session of SKY generates global brain rhythm dominantly with high-frequency cerebral activation and initiates appropriate inter hemispheric synchronization in brain rhythms as state effects. This suggests that SKY leads to better attention, memory, emotional and autonomic control along with enhanced cognitive functions, which further improve physical and mental well-being [24]. SKY can improve working memory capacity by changing the brain rhythms so that energy is utilized efficiently in performing a task [27]. Research also indicates that prolonged meditation training improves parietal-occipital EEG gamma activity during sleep. This change in gamma activity is required for NREM sleep indicating a better quality [38]. Though the brain structure was not studied here among SKY practitioners, the effect is similar resulting in better sleep quality after prolonged SKY practice.

Vagal nerve stimulation and parasympathetic activity regulation post SKY are key factors for improving sleep quality among individuals [21]. A two-way correlation is noticed between sleep and psychiatric disorders. Therapeutic potential of SKY in treating mild to moderate depression and anxiety has been studied in the past with a positive impact experienced by $74.6 \%$ of the population. Improvement in mental morbidities can lead to improvement of sleep quality 
[14]. On the other hand, sleep difficulties are a causal factor for many psychotic experiences as noted by Freeman et al. [39].

This was a pilot study to understand the benefits of SKY on sleep, and there were a few limitations. The key limitation includes the lack of a control group and adoption of convenience sampling study design. A large sample size of 473 participants is beneficial with respect to the accuracy of the result; however, the sample size to reduced significantly on day 40 assessment. Besides the stress levels commodities, BMI or health status of the participant before the intervention was not assessed. The wakeful cycle or any routine activity of these participants have also not been studied.

Having said that, these results serve as important data for further clinical studies. The SKY intervention is shown to be feasible and reproducible. SKY has also been delivered in different clinical contexts as demonstrated by prior research studies.

\section{Conclusion}

This is a unique study demonstrating the positive impact of SKY yogic breathing technique on the sleep quality in healthy adults, and is indicative of SKY's possible beneficial role in treating psychological disorders such as insomnia. Improvement was noticed across the study population irrespective of gender or age. However, sustained benefits of SKY were most observed with daily practice. Therefore, incorporating few minutes of holistic breathing in daily lifestyle can enhance sleep quality, promote good health and enhance the quality of life.

Supplementary Information The online version contains supplementary material available at https://doi.org/10.1007/s41782-021-00146-4.

Open Access This article is licensed under a Creative Commons Attribution 4.0 International License, which permits use, sharing, adaptation, distribution and reproduction in any medium or format, as long as you give appropriate credit to the original author(s) and the source, provide a link to the Creative Commons licence, and indicate if changes were made. The images or other third party material in this article are included in the article's Creative Commons licence, unless indicated otherwise in a credit line to the material. If material is not included in the article's Creative Commons licence and your intended use is not permitted by statutory regulation or exceeds the permitted use, you will need to obtain permission directly from the copyright holder. To view a copy of this licence, visit http://creativecommons.org/licenses/by/4.0/.

\section{References}

1. Zielinski MR, McKenna JT, McCarley RW. Functions and mechanisms of sleep. AIMS Neurosci. 2016;3(1):67-104. https://doi. org/10.3934/Neuroscience.2016.1.67.
2. Zee PC. Sleep and health. Arch Intern Med. 2006;166(16):1686. https://doi.org/10.1001/archinte.166.16.1686.

3. The global pursuit of better sleep health, 2019. Philips. https:// www.usa.philips.com/c-dam/b2c/master/experience/smartsleep/ world-sleep-day/2019/2019-philips-world-sleep-day-survey-resul ts.pdf (2019)

4. Stranges S, Tigbe W, Gómez-Olivé FX, Thorogood M, Kandala N-B. Sleep problems: an emerging global epidemic? Findings from the indepth WHO-SAGE study among more than 40,000 older adults from 8 countries across Africa and Asia. Sleep. 2010;35(8):1173-81. https://doi.org/10.5665/sleep.

5. Stone KL, Xiao Q. Impact of poor sleep on physical and mental health in older women. Sleep Med Clin. 2018;13(3):457-65. https://doi.org/10.1016/j.jsmc.2018.04.012.

6. Homma I, Masaoka Y. Breathing rhythms and emotions. Exp Physiol. 2008;93(9):1011-21. https://doi.org/10.1113/expph ysiol.2008.042424.

7. Zaccaro A, Piarulli A, Laurino M, Garbella E, Menicucci D, Neri B, Gemignani A. How breath-control can change your life: a systematic review on psycho-physiological correlates of slow breathing. Front Hum Neurosci. 2018. https://doi.org/10.3389/ fnhum.2018.00353.

8. Kjellgren A, Bood SA, Axelsson K, Norlander T, Saatcioglu F. Wellness through a comprehensive yogic breathing program-a controlled pilot trial. BMC Complement Altern Med. 2007;7:43. https://doi.org/10.1186/1472-6882-7-43.

9. Brown RP, Gerbarg PL. Sudarshan Kriya yogic breathing in the treatment of stress, anxiety, and depression: part I-neurophysiologic model. J Altern Complement Med. 2005;11(1):189-201. https://doi.org/10.1089/acm.2005.11.189 (PubMed: 15750381).

10. Doria S, de Vuono A, Sanlorenzo R, Irtelli F, Mencacci C. Antianxiety efficacy of Sudarshan Kriya Yoga in general anxiety disorder: A multicomponent, yoga based, breath intervention program for patients suffering from generalized anxiety disorder with or without comorbidities. J Affect Disord. 2015;184:310-7. https://doi.org/10.1016/j.jad.2015.06.011.

11. Katzman MA, Vermani M, Gerbarg PL, Brown RP, Iorio C, Davis M, et al. A multicomponent yoga-based, breath intervention program as an adjunctive treatment in patients suffering from generalized anxiety disorder with or without comorbidities. Int J Yoga. 2012;5:57-65. https://doi.org/10.4103/09736131.91716 (PMCID:PMC3276935 PubMed: 22346068).

12. Agte VV, Chiplonkar SA. Sudarshan Kriya yoga for improving antioxidant status and reducing anxiety in adults. Altern Complement Ther. 2008;14(2):96-100. https://doi.org/10.1089/act. 2008.14204.

13. Subramanian S, Elango T, Malligarjunan H, Kochupillai V, Dayalan H. Role of sudarshan kriya and pranayam on lipid profile and blood cell parameters during exam stress: a randomized controlled trial. Int J Yoga. 2012;5:21-7. https://doi.org/ 10.4103/0973-6131.91702 (PMCID: PMC3276928 PubMed: 22346062).

14. Hamilton-West K, Pellatt-Higgins T, Sharief F. Evaluation of a Sudarshan Kriya Yoga (SKY) based breath intervention for patients with mild-to-moderate depression and anxiety disorders. Prim Healthc Res Dev. 2019;20:e73. https://doi.org/10.1017/ S1463423619000045.

15. Seppälä EM, Nitschke JB, Tudorascu DL, et al. Breathing-based meditation decreases posttraumatic stress disorder symptoms in U.S. military veterans: a randomized controlled longitudinal study. J Trauma Stress. 2014;27(4):397-405. https://doi.org/10. 1002/jts. 21936 (PMCID: PMC4309518 PubMed: 25158633).

16. Descilo T, Vedamurtachar A, Gerbarg PL, Nagaraja D, Gangadhar BN, Damodaran B, et al. Effects of a yoga breath intervention alone and in combination with an exposure therapy for posttraumatic stress disorder and depression in survivors of the 2004 
South-East Asia tsunami. Acta Psychiatr Scand. 2010;121:289300. https://doi.org/10.1111/j.1600-0447.2009.01466.x (PubMed: 19694633).

17. Gootjes L, Franken IH, Van Strien JW. Cognitive emotion regulation in yogic meditative practitioners. J Psychophysiol. 2011;25:87-94. https://doi.org/10.1027/0269-8803/a000043.

18. Ghahremani DG, Oh EY, Dean AC, Mouzakis K, Wilson KD, London ED. Effects of the Youth Empowerment Seminar on impulsive behavior in adolescents. J Adolesc Health. 2013;53(1):139-41. https://doi.org/10.1016/j.jadohealth.2013. 02.010 (PubMed: 23601502).

19. Seppälä EM, Bradley C, Moeller J, Harouni L, Nandamudi D, Brackett MA. Promoting mental health and psychological thriving in university students: a randomized controlled trial of three well-being interventions. Front Psychiatry. 2020;11:590. https:// doi.org/10.3389/fpsyt.2020.00590.

20. Goldstein MR, Lewin RK, Allen JJB. Improvements in well-being and cardiac metrics of stress following a yogic breathing workshop: randomized controlled trial with active comparison. J Am Coll Health. 2020. https://doi.org/10.1080/07448481.2020.17818 67.

21. Goldstein MR, Lewis GF, Newman R, Brown JM, Bobashev G, Kilpatrick L, Seppälä EM, Fishbein DH, Meleth S. Improvements in well-being and vagal tone following a yogic breathing-based life skills workshop in young adults: two open-trial pilot studies. Int J Yoga. 2016;9(1):20-6. https://doi.org/10.4103/0973-6131. 171718

22. Toschi-Dias E, Tobaldini E, Solbiati M, et al. Sudarshan Kriya Yoga improves cardiac autonomic control in patients with anxietydepression disorders. J Affect Disord. 2017;214:74-80. https:// doi.org/10.1016/j.jad.2017.03.017.

23. Somwanshi SD, Handergulle SM, Adgaonkar BD, Kolpe DV. Effect of Sudarshan Kriya Yoga on cardiorespiratory parameters. Int J Recent Trends Sci Tech. 2013;8(1):62-6 (Corpus ID: 28183769)

24. Bhaskar L, Kharya C, Deepak KK, Kochupillai V. Assessment of cardiac autonomic tone following long Sudarshan Kriya Yoga in art of living practitioners. J Altern Complement Med. 2017;23(9):705-12. https://doi.org/10.1089/acm.2016.0391.

25. Kharya C, Gupta V, Deepak KK, Sagar R, Upadhyav A, Kochupillai V, Anand S. Effect of controlled breathing exercises on the psychological status and the cardiac autonomic tone: Sudarshan Kriya and Prana-Yoga. Indian J Physiol Pharmacol. 2014;58(3):211-21 (PMID: 25906603).

26. Chandra S, Jaiswal AK, Singh R, Jha D, Mittal AP. Mental stress: neurophysiology and its regulation by Sudarshan Kriya Yoga. Int J Yoga. 2017;10(2):67-72. https://doi.org/10.4103/0973-6131. 205508.

27. Chandra S, Sharma G, Mittal AP, Jha D. Effect of Sudarshan Kriya (meditation) on gamma, alpha, and theta rhythm during working memory task. Int J Yoga. 2016;9(1):72-6. https://doi. org/10.4103/0973-6131.171715.

28. Chandra S, Sharma G, Sharma M, Jha D, Mittal AP. Workload regulation by Sudarshan Kriya: an EEG and ECG perspective. Brain Inform. 2017;4(1):13-25. https://doi.org/10.1007/ s40708-016-0055-1.
29. Sulekha S, Thennarasu K, Vedamjurthachar A, Raju TR, Kutty BM. Evaluation of sleep architecture in practitioners of Sudarshan Kriya yoga and Vipassana meditation. Sleep Biol Rhythms. 2006;4(3):207-14. https://doi.org/10.1111/j.1479-8425.2006. 00233.x.

30. Kishida M, Mama SK, Larkey LK, Elavsky S. "Yoga resets my inner peace barometer": a qualitative study illuminating the pathways of how yoga impacts one's relationship to oneself and to others. Complement Thern Med. 2018;40:215-21. https://doi.org/ 10.1016/j.ctim.2017.10.002.

31. Vera FM, Manzaneque JM, Maldonado EF, Carranque GA, Rodriguez FM, Blanca MJ, Morell M. Sleep Quality and hormonal modulation in long-term yoga practitioners. Bio Psych. 2009;81(3):164-8. https://doi.org/10.1016/j.biopsycho.2009.03. 008.

32. Koffel E, Bramoweth AD, Ulmer CS. Increasing access to and utilization of cognitive behavioral therapy for insomnia (CBT-I): a narrative review. J Gen Intern Med. 2018;33(6):955-62. https:// doi.org/10.1007/s11606-018-4390-1.

33. Rusch HL, Rosario M, Levison LM, Olivera A, Livingston WS Wu T, Gill JM. The effect of mindfulness meditation on sleep quality: a systematic review and meta-analysis of randomized controlled trials. Ann NY Acad Sci. 2019;1445(1):5-16. https:// doi.org/10.1111/nyas.13996.

34. Nagendra RP, Maruthai N, Kutty BM. Meditation and its regulatory role on sleep. Mini review article. Front Neuro. 2012;3:1-4. https://doi.org/10.3389/fneur.2012.00054.

35. Neuendorf R, Wahbeh H, Chamine I, Yu J, Hutchison K, Oken, BS. The effects of mind-body interventions on sleep quality: a systematic review. Evid Based Complement Alternat Med. 2015. Article ID 902708. https://doi.org/10.1155/2015/902708

36. Dzierzewski JM, Dautovich N, Ravyts S. Sleep and cognition in the older adult. Sleep Med Clin. 2018;13(1):93-106. https://doi. org/10.1016/j.jsmc.2017.09.009.

37. Nupur R, Sumeet I, Shashank K, Divya K. Effect of Sudarshan Kriya Yoga and associated meditation techniques on the connection and sensitivity towards environment. Int J Innov Res Sci Eng Tech. 2018;7(12):11899-909. https://doi.org/10.15680/IJIRSET. 2018.0712038.

38. Dentico D, Ferrarelli F, Riedner BA, Smith R, Zennig C, Lutz A, Tononi G, Davidson RJ. Short meditation trainings enhance non-REM sleep low-frequency oscillations. PLoS ONE. 2016;11(2):e0148961. https://doi.org/10.1371/journal.pone.01489 61.

39. Freeman D, Sheaves B, Goodwin GM, Yu LM, Nickless A, Harrison PJ, Emsley R, Luik AI, Foster RG, Wadekar V, Hinds C. The effects of improving sleep on mental health (OASIS): a randomised controlled trial with mediation analysis. Lancet Psychiatry. 2017;4(10):749-58. https://doi.org/10.1016/S2215-0366(17) 30328-0.

Publisher's Note Springer Nature remains neutral with regard to jurisdictional claims in published maps and institutional affiliations. 